\title{
Impact of FIFRELIN input parameters on fission observables
}

\author{
Abdelaziz Chebboubi $^{1, \star}$, Olivier Litaize ${ }^{1}$, and Olivier Serot ${ }^{1}$ \\ ${ }^{1}$ CEA, DEN, DER, SPRC, Cadarache, Physics Studies Laboratory, F-13108 Saint-Paul-lès-Durance, France
}

\begin{abstract}
Evaluated nuclear data are essential for nuclear reactor studies. In order to significantly improve the precision of nuclear data, more and more fundamental fission models are used in the evaluation processing. Therefore, tests of fission models become a central issue. In this framework, FIFRELIN (FIssion Fragments Evaporation Leading to an Investigation of Nuclear data) is a Monte Carlo code developed in order to modelize fission fragments de-excitation through the emission of neutrons, $\gamma$ and conversion $e^{-}$. To be performed, a FIFRELIN calculation relies on several models such as gamma strength function and nuclear level density and of more empirical hypothesis such as total excitation energy repartition or angular momentum given by the fission reaction. Moreover, pre-emission mass yield and kinetic energy distribution per mass are necessary to process the simulation. A set of five free parameters are chosen to reproduce a target observable. Often this observable corresponds to the mean neutron multiplicity for heavy and light fragment. In this work, the impact of the set of parameters on different output observables (neutron emission probability, neutron multiplicity as function of the fission fragment mass) is investigated.
\end{abstract}

\section{Introduction}

Nuclear reactor simulations rely more and more on complex multi-physics modelling. In order to reach target precision, it is necessary to improve the accuracy of fission data in the actinide region. Nowadays, two complementary paths are explored. The first one comes from the experimental side. New instruments and analysis are developed with new measured observables. The second one comes from the theoretical side. New models such as FIFRELIN aims to improve the predictibility of models by looking simultaneously at multiple observables.

\subsection{Description of FIFRELIN}

FIFRELIN is a Monte Carlo code developed at CEA Cadarache [1-3]. It focuses on the emission of prompt particles $\left(n, \gamma, e^{-}\right)$from fission fragments. Most fission observables such as neutron multiplicity, kinetic energy distribution of fission fragments among others can be calculated. The code requires input files (generally derived from experimental data) as well as models to properly reproduce the de-excitation path of fission fragments. The code is a two step process :

\footnotetext{
^e-mail: abdelhazize.chebboubi@ cea.fr
} 
- After being created at the scission point and accelerated, fission fragments are characterized by their kinetic energy KE, excitation energy $E^{*}$, total angular momentum and parity $J^{\pi}$, mass $A$ and nuclear charge $Z$. These observables are sampled from experimental data (A,KE) or from models (Wahl's model [4] for Z) or from more simple distribution $(J, \pi)$. Concerning this last point, two free parameters $\sigma_{L}$ and $\sigma_{H}$, which correspond to the mean angular momentum for light and heavy fission fragment region respectively, are necessary :

$$
P(J) \propto(2 J+1) \exp \left(-\frac{(J+1 / 2)^{2}}{2 \sigma^{2}}\right),
$$

with $P(J)$ the probability distribution for $\mathrm{J}$ with $\sigma$ also called the spin cutoff. Finally, $E^{*}$ is calculated from energy conservation law:

$$
\mathrm{TXE}=Q-\mathrm{TKE}=E_{L}^{*}+E_{H}^{*}+E_{L}^{\mathrm{rot}}+E_{H}^{\mathrm{rot}},
$$

with TXE the total available excitation energy, $Q$ the energy released by the fission process, TKE the total kinetic energy and $E^{\text {rot }}$ the collective rotational energy for both light $(\mathrm{L})$ and heavy $(\mathrm{H})$ fission fragment region. By definition the rotational energy is expressed as:

$$
E^{\mathrm{rot}}=\frac{\hbar^{2} J(J+1)}{2 I}
$$

with $\mathcal{I}$ the fission fragment moment of inertia. By default, $\mathcal{I}=k \times \mathcal{I}_{\text {rigid }}$ with $k$ a free parameter and $\mathcal{I}_{\text {rigid }}$ the moment of inertia of a rigid spheroid. The repartition of the remaining excitation energy $E_{L}^{*}+E_{H}^{*}$ is achieved through a phemenological temperature ratio law $\mathrm{RT}(A)=T_{L} / T_{H}$ :

$$
\frac{E_{L}^{*}}{E_{H}^{*}}=\frac{a_{L} \mathrm{RT}^{2}}{a_{H}},
$$

where $a$ stands for the level density parameter. Figure 1 (left) shows the behaviour of the temperature ratio law. Two additional parameters, $\mathrm{RT}_{\min }$ and $\mathrm{RT}_{\max }$, are required in order to control the repartition of the available excitation energy.

- Once fission fragments are sampled with specific mass, nuclear charge, kinetic energy and excitation state, next step is to de-excite the fragment until the ground state is reached, or a $\beta$ decay occurs. To do so, the nuclear structure scheme for each fission fragment is built. This scheme is mixing experimental data and those derived from models. In concrete terms, above a cut-off energy $\left(E_{\text {cut-off }}^{\mathrm{RIPL}}\right)$, the discrete experimental nuclear scheme coming from the RIPL-3 database [5] is completed with discrete levels with a model of level density (here CGCM [6]) up to a second cut-off energy $\left(E_{\text {bin }}\right)$. Above this energy, the nuclear level scheme is described as a continuum. This sketch is shown on Fig. 1 (right). The last part of the code is to determine the de-excitation path from the initial state previously sampled. A state can decrease by emitting neutron, $\gamma$ or $e^{-}$. The associated probabilities are calculated through:

- transmission coefficients which were determined by an external code (TALYS/ECIS [7]) and depend on optical model parametrizations (case of neutron)

- models of $\gamma$ strength function (here EGLO [8]) (case of $\gamma$ )

- experimental data (case of $\gamma$ and $e^{-}$)

Note that FIFRELIN uses the notion of Nuclear Realization developed in [9] and extended to neutron $/ \gamma$ coupled emission [3]. 

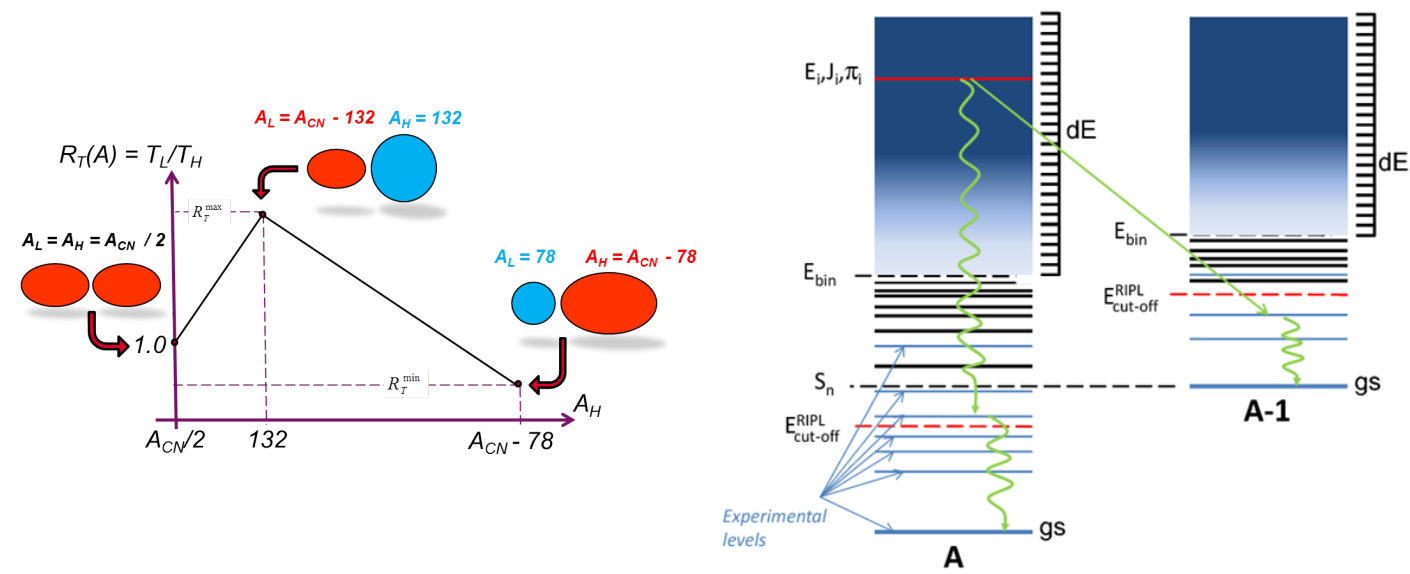

Figure 1. Repartition of the total excitation energy through a temperature ratio law with two free parameters: $\mathrm{RT}_{\min }$ and $\mathrm{RT}_{\max }$ (left). Illustration of the FIFRELIN procedure to complete the nuclear structure scheme from experimental information to the continuum (right).

\section{Free parameters and target observables for spontaneous fission of ${ }^{252} \mathbf{C f}$}

As described above, FIFRELIN has five free parameters: $\mathrm{RT}_{\min }, \mathrm{RT}_{\max }, \sigma_{L}, \sigma_{H}$ and $k$. In this work, $k$ was fixed to 1 . The other parameters are adjusted in order to reproduce a target observable which is supposed to be well-known. In the case of ${ }^{252} \mathrm{Cf}(\mathrm{sf})$, the mean neutron multiplicity $(\bar{v})$ together with the mean neutron multiplicity for light $\left(\overline{v_{L}}\right)$ and heavy $\left(\overline{v_{H}}\right)$ fission fragment groups are considered as standard values. A first set of free parameters is determined in order to reproduce accurately those three target observables. All the following results associated to this set are labelled "Ref".

Recently, the measurement of prompt fission gamma spectrum by Oberstedt et al. [10] give access to the $\gamma$ multiplicity $\left(\overline{M_{\gamma}}\right)$, between $100 \mathrm{keV}$ and $7 \mathrm{MeV}$ for a time windows of $3 \mathrm{~ns}$. 528 sets of free parameters were calculated and compared to $\bar{v}$ and $\overline{M_{\gamma}}$ only. For each $\left(\sigma_{L}, \sigma_{H}\right)$ fixed value, 16 $\left(\mathrm{RT}_{\min }, \mathrm{RT}_{\max }\right)$ values are calculated, where

- $\mathrm{RT}_{\min }$ goes from 0.2 to 0.8 by step of 0.2

- $\mathrm{RT}_{\max }$ goes from 1.1 to 1.9 by step of 0.2

Figure 2 shows the difference between simulation and experimental data:

$$
\Delta=\frac{\overline{v^{\exp }}-\overline{v^{\mathrm{FIF}}}}{\sigma_{\overline{v^{\exp }}}^{2}+\sigma_{\overline{v^{\mathrm{FF}}}}^{2}}+\frac{\overline{M^{\exp }}-\overline{M^{\mathrm{FIF}}}}{\sigma_{\overline{M^{\exp }}}^{2}+\sigma_{\overline{M^{\mathrm{FIF}}}}^{2}}
$$

The RED color corresponds to $\Delta>100$. From all the combinations, only three were selected in order to run simulations with more statistics ( 1 million of fission events compared with 7000 events for the 528 runs). Table 1 synthesizes the three different selected simulations as well as the "Ref" case. The following part of this article will compare, for various fission observables, these four different simulations including the reference case. It is clear from Fig. 2 that very high and very low values of $\sigma_{L}, \sigma_{H}$ can't be used to reproduce the fission observables (red blocs). 


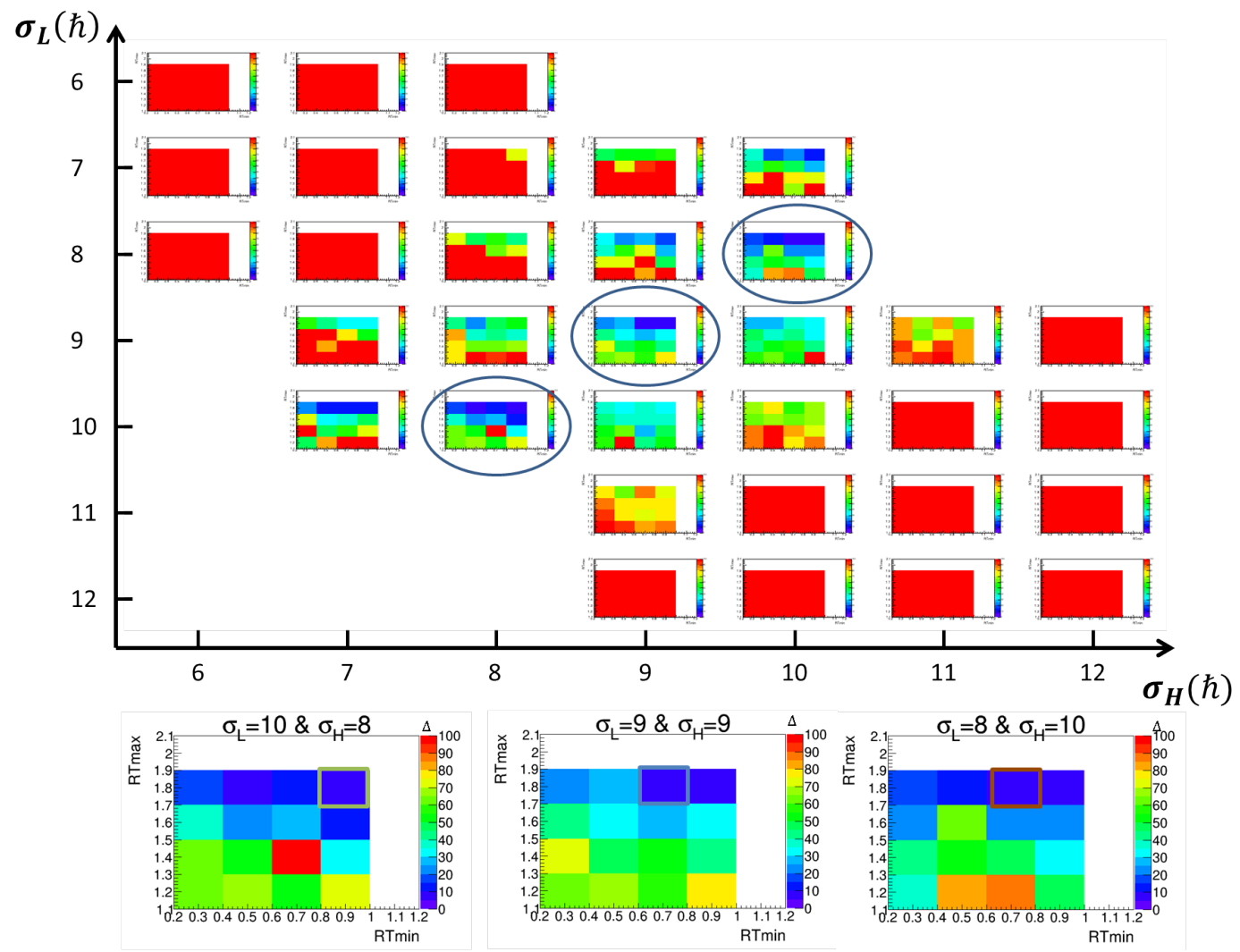

Figure 2. Comparison of $\bar{v}$ and $\overline{M_{\gamma}}$ from simulations and experimental data. 528 simulations of input parameters $\left(\mathrm{RT}_{\min }, \mathrm{RT}_{\max }, \sigma_{L}, \sigma_{H}\right)$ were performed. The Warmer the color, the bigger is the difference compared to experimental data. The best cases are plotted in the lower part of the figure.

Table 1. Input parameters for the four simulations. Target observables are computed along with associated experimental value.

\begin{tabular}{|c|c|c|c|c|c|c|c|c|}
\hline Simulation & $\mathrm{RT}_{\text {min }}$ & $\mathrm{RT}_{\text {max }}$ & $\sigma_{L}$ & $\sigma_{H}$ & $\bar{v}$ & $\overline{v_{L}}$ & $\overline{v_{H}}$ & $\overline{M_{\gamma}}$ \\
\hline Ref & 0.35 & 1.41 & 10.5 & 11 & 3.752 & 2.070 & 1.682 & 10.58 \\
\hline Up & 0.8 & 1.7 & 10 & 8 & 3.792 & 2.530 & 1.261 & 8.97 \\
\hline Middle & 0.6 & 1.7 & 9 & 9 & 3.812 & 2.463 & 1.349 & 8.94 \\
\hline Down & 0.6 & 1.7 & 8 & 10 & 3.811 & 2.454 & 1.357 & 8.95 \\
\hline Exp & & & & & $\begin{array}{l}3.757 \pm \\
0.01[11]\end{array}$ & $2.051[12]$ & 1.698 [12] & $\begin{array}{lr}8.29 \pm \\
0.13[10]\end{array}$ \\
\hline
\end{tabular}

\section{Consequences on fission observables}

In this work, the comparison between the four simulations and experimental data was achieved for prompt neutron and $\gamma$ observables. 

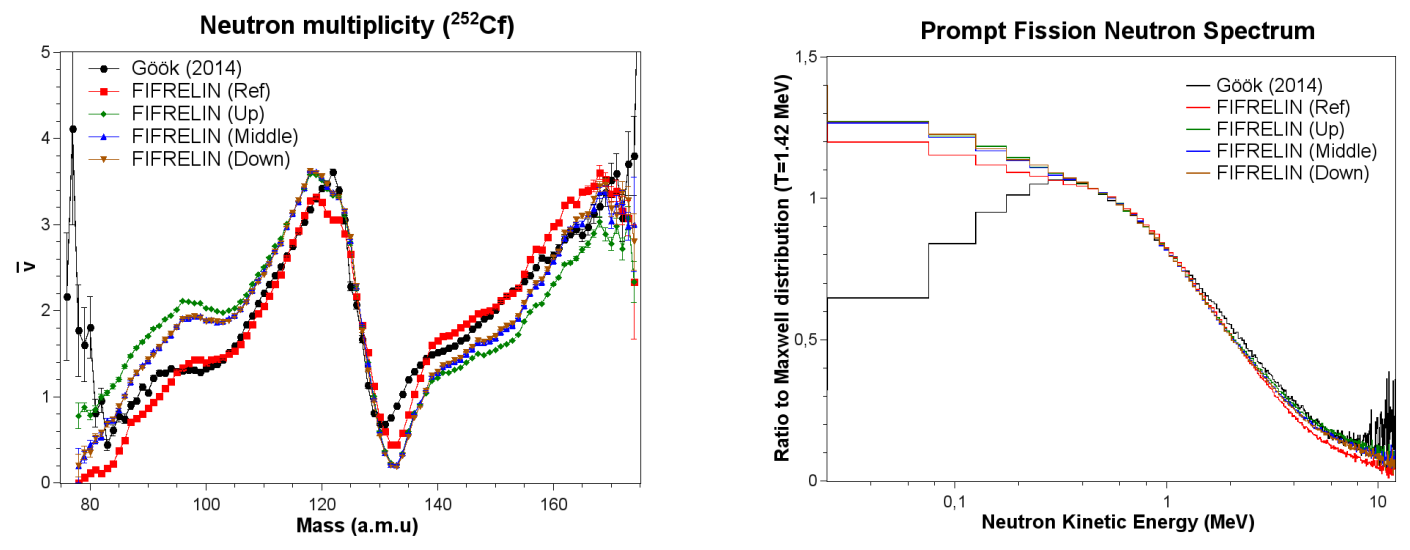

Figure 3. Comparison of the sawtooth (left) and PFNS (right) for the four simulations and experimental data [13].
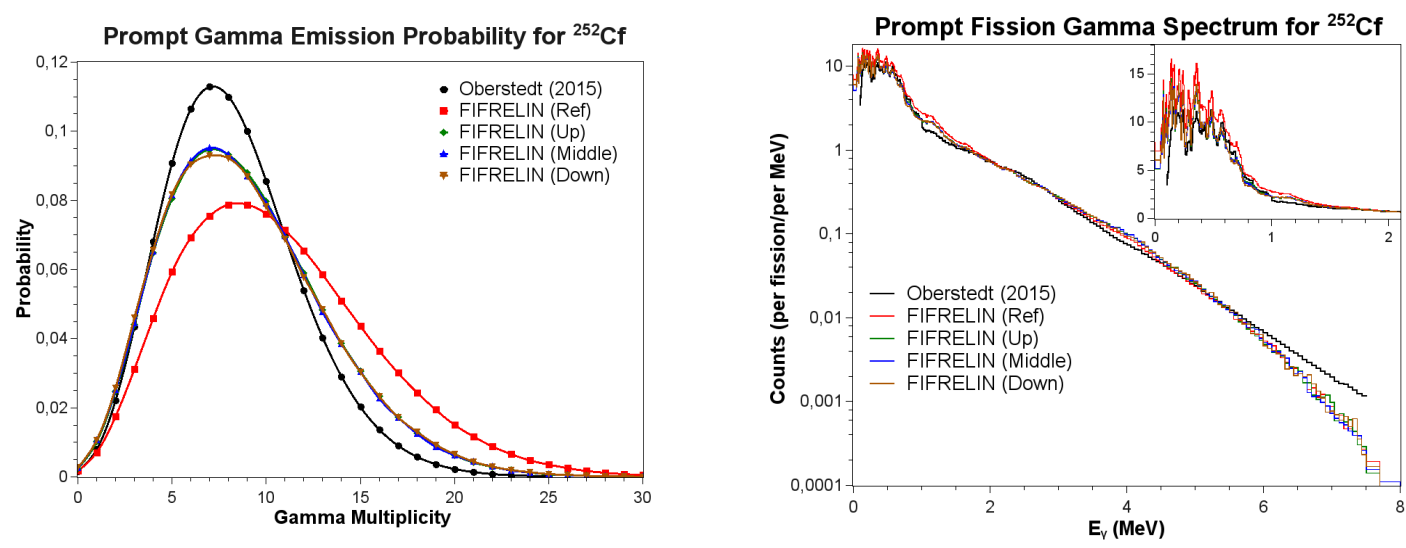

Figure 4. Comparison of the gamma multiplicity distribution (left) and PFGS (right) for the four simulations and experimental data [10].

- Case of neutron observables: Fig. 3 shows the results for the neutron multiplicity as function of the fission fragment mass (left) and the associated spectrum (right). Experimental data are plotted in black, while red color corresponds to the "Ref" simulation, green to the "Up" simulation, blue to the "Middle" simulation and brown to the "Down" simulation. The "Ref" reproduce quite well the sawtooth behaviour of the neutron multiplicity. While $\bar{v}$ is conserved (around 3.8 instead of 3.76) the partial neutron multiplicity are over(under) estimated for the light(heavy) fission fragment group, for the last three simulations. Note that apart from a shift, some structures are more important. Finally it is interessing to see that "Middle" and "Down" simulations are almost identical. Therefore this observable is really sensitive to the ratio $\mathrm{RT}_{\max } / \mathrm{RT}_{\min }$. However, one has to remember that even though the repartition of neutron multiplicity to light and heavy group is mainly governed by RT parameters, the impact of $\sigma_{L}$ and $\sigma_{H}$ is not negligible as shown by Fig. 2.

Concerning the prompt fission neutron spectrum a slight difference is observed between the three last simulations and the "Ref" one. This observable seems to be mainly governed by models of level 
density and neutron transmission coefficients. Indeed, since all simulations have almost the same $\bar{v}$, the quasi-unchanged spectrum means that the initial fission fragment states, which are directly related to the input parameters, have few impact on this observable.

- Case of gamma observables: Fig. 4 shows the results for the $\gamma$ multiplicity distribution (left) and the spectrum (right). The last three simulations (with lower $\sigma_{L}$ or $\sigma_{H}$ than the "Ref" simulation) improve the comparison with experimental data. However, an overestimation of low energy $\gamma$-rays and high multiplicity of the calculations are still present. Both effects can be closely related. For instance, at high excitation energy, models of $\gamma$ strength function seem to favor the emission of multiple low energy $\gamma$-rays over the emission of one or two high energy $\gamma$-rays. New development to reduce the multiplicity of low energy $\gamma$-rays is under progress.

\section{Conclusion and perspectives}

FIFRELIN is a Monte Carlo code which fully describes fission fragment characteristics. It involves different models to simulate a fission de-excitation and the entry zone given by the fission process. Both experimental and calculated data are used. Impact of input parameters on fission observables were highlighted. It gives insight on the current limits of the code. New developments are under progress in order to better control the sensitivy on the input parameters and improve the prediction power of FIFRELIN.

\section{References}

[1] O. Litaize, O. Serot, Phys. Rev. C 82, 054616 (2010)

[2] O. Litaize et al., EPJ A 51, 1 (2015)

[3] D. Regnier et al., Comput. Phys. Commun. 201, 19 (2016)

[4] A.C. Wahl, At. Data. Nucl. Data Tables 39, 1 (1988)

[5] R. Capote et al., Nucl. Data Sheets 110, 3107 (2009)

[6] A. Gilbert, A.G.W. Cameron, Can. J. of Phys. 43, 1446 (1965)

[7] A. Koning et al., TALYS-1.0 (EDP Sciences, 2007), pp. 211-214

[8] J. Kopecky, M. Uhl, Phys. Rev. C 41, 1941 (1990)

[9] F. Bečvář, Nucl. Instrum. Meth. A 417, 434 (1998)

[10] A. Oberstedt et al., Phys. Rev. C 92, 014618 (2015)

[11] P. Santi, M. Miller, Nucl. Sci. Eng. 160 (2008)

[12] A. Vorobyev et al., in AIP Conf. Proc. (AIP, 2005), Vol. 769, pp. 613-616

[13] A. Göök et al., Phys. Procedia 64, 190 (2015) 